\title{
Long QT testing: implications for complex diagnosis in personalized medicine
}

"...10\% of long QT syndrome gene carriers are asymptomatic and at risk of sudden death."

Long QT syndrome (LQTS) is a cardiac condition characterized by prolonged QT time on the ECG, representing delayed cardiac repolarization and a propensity for ventricular tachycardia. The disease has an estimated prevalence of 1 in 2500 and a clinical presentation that includes sudden death and syncope. Mutations in 12 genes have been demonstrated to cause the disease (LQTS1-12); all the genes code for either cardiac ion channels or proteins interacting with these channels [1].

Presently, LQTS genetic testing, performed by most laboratories, provides comprehensive open reading frame/splice site mutational analysis of the five most frequently affected LQTS-causing genes, that is, KCNQ1, KCNH2, SCN5A, $K C N E 1$ and $K C N E 2$, via high-throughput DNA sequencing $[2,3]$. A disease-causing mutation can be identified in $50-70 \%$ of symptomatic LQTS cases and cascade screening is routinely performed in affected families [2,3]. This is important as $10 \%$ of LQTS gene carriers are asymptomatic and at risk of sudden death [4]; LQTS has been estimated to be responsible for up to $10 \%$ of sudden infant death syndrome and 5\% of sudden adult death syndrome cases [5]. There is no doubt that the real evidence-based value of LQTS testing at present relates to cascade screening in families of people with symptomatic disease, exemplified by sudden death cases with an identified presumed disease-causing mutation. However, it should be noted that the diagnosis of LQTS in most cases is a clinical one - genetic testing can not replace careful clinical examination as a negative mutation finding does not rule out the condition.

The genetics of LQTS is complicated, which is exemplified by the problems in ascertaining the clinical significance of novel mutations. These problems are not merely a consequence of allelic heterogeneity, but also due to the fact that most identified mutations are private, that is, specific for the examined family [1] and approximately 5\% of LQTS cases are compound heterozygous [6,7]. The existence of many rare polymorphisms (and genetic modifiers) in the involved genes further complicates the genetic assessment of LQTS patients [8]. Many disease-causing mutations result in changes in the function of ion channels, for example, gating properties, and require highly specialized electrophysiological analysis for their characterization [1]. Such an analysis is not readily available and certainly not within a reasonable timeframe. Finally, small family sizes often preclude a certain assessment of disease segregation with the mutation in the pedigree. In reality, most of the mutations described in the literature as 'disease causing' have not been confirmed to be so in sensu stricto.

For the individual patient, several benefits can be noted if a disease-causing mutation can be found. First, prognostic information can occasionally be gained from knowing the affected gene [9] or the location of the mutation within a specific gene [10]. Second, if the person decides to have children they may be analyzed genetically at birth, or for that matter prenatally, and necessary prophylactic treatment can be instituted prior to occurrence of symptoms, reducing the need for extensive clinical investigations in early childhood. Third, knowledge of the gene involved may also aid in the identification of behavioral risk factors and enable a more precise recommendation of lifestyle changes. Mutations in some genes have been demonstrated to be associated with specific arrhythmia-precipitating factors, for example, physical activity in KCNQ1 mutations and emotional distress in $\mathrm{KCNH} 2$ mutations [1]. Thus, the identification of a disease-causing mutation may obviate the need for indiscriminate banning of physical activities. Finally, as the effect of treatment differs between affected genes and mutations in some genes - for example, SCN5A - exhibit complex phenotypes, genotyping may assist in defining optimal treatment or suggest further clinical testing [11]. The latter

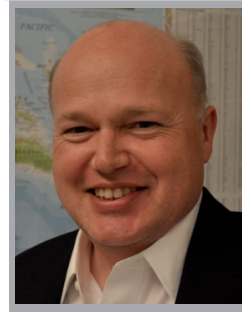

Michael Christiansen

Author for correspondence:

Department of Clinical

Biochemistry and Immunology,

Statens Serum Institut,

5 Artillerivej DK 2300 S,

Copenhagen, Denmark

Tel.: +4532683657

Fax: +4532688265

mic@ssi.dk

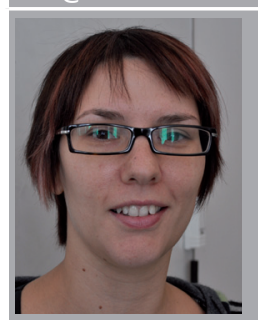

Paula L Hedley

Department of Clinical

Biochemistry and Immunology,

Statens Serum Institut,

Copenhagen, Denmark

and

Department of Biomedical

Sciences, University of

Stellenbosch, Cape Town,

South Africa

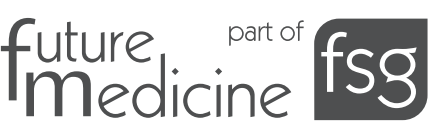


has recently been demonstrated to be the case for mutations in SCN5A, where some mutations present phenotypically as LQTS or atrial fibrillation, but upon flecainide stimulation reveal a Brugada phenotype that may require the insertion of an intracardiac defibrillator unit for optimal prophylaxis against sudden death [12].

\section{"There is no doubt that the real evidence-based value of long QT syndrome testing at present relates to cascade screening in families of people with symptomatic disease..."}

Genetic testing for LQTS also raises problems for the individual. First, if genetic testing is carried out with individuals who have no symptoms or family history of LQTS symptoms, there is a considerable risk of identifying insignificant novel variants and misinterpreting them as being associated with LQTS in its 'forme fruste' presentation. Genetic testing should be reserved for people with clinical LQTS or relatives of patients with LQTS. Thus, screening for LQTS only through mutation screening in LQTS genes is not possible and is likely to be detrimental because it results in the introduction of non-evidence-based morbidity. Furthermore, most of the available information on prognosis of different mutations, treatment results and precipitating factors is based on a phenotyping of variable quality, performed on a limited subset of all genetic variants in particular genes, reducing the evidence base of the clinical inferences - particularly so in the individual case. Even in patients with clinical LQTS, the interpretation of finding a mutation has to be taken with caution, as the wide range of clinical severity of the same mutation, even within a family, makes it difficult to benefit from the mutation identification in the individual.

"Genetic testing should be reserved for
people with clinical long QT syndrome or
relatives of patients with long QT syndrome."

It is the authors' opinion that several precautions can be taken to avoid a faulty interpretation of genetic testing for LQTS. The mutation screens should be expanded to incorporate all 12 genes, as this will reduce the risk of overlooking mutations in rarely affected genes as well as compound heterozygosity. Furthermore, the clinical inference of finding a mutation should be made with care, acknowledging the frequently weak knowledge base of statements on prognostic significance and phenotypical characteristics. However, these precautions should be weighed against the clinical situation of the affected family. This means that the significance accorded to a novel putative disease-causing mutation should be measured against the clinical severity of the disease in the family.

There is no doubt that the pros and cons of LQTS testing is very much in favor of the pros in cases where the person examined has LQTS or is at risk of having it owing to the family history. On the contrary, the cons are very important in cases where LQTS diagnosis has not been established.

\section{"Long QT syndrome illustrates the problems associated with the identification of disease- causing mutations in individual patients when a plethora of genes may be involved, occasionally simultaneously."}

It is noteworthy that LQTS has turned out to be far more complicated than initially thought; the complexity, that is, the increasing number of genes to be analyzed when testing, is being overcome with the advances in sequencing technology. However, this does not solve the problems associated with the interpretation of the test results and the entailing clinical recommendations to the individual gene carrier. In addition, this simple autosomal dominant Mendelian disorder has turned into a polygenic disorder. Future research will certainly solve some of the problems associated with LQTS testing; the possibility of massive parallel sequencing of all exons in the genome [13] will increase the success rate of identification of putative disease-causing mutations but, at the same time, increase the need to refine the methods of eliminating insignificant rare variants and functional assessment of ion channel function. It remains an open question as to whether the dramatically increased sequence information will increase the diagnostic efficiency or increase the confusion with respect to the significance of genetic variants.

Long QT syndrome illustrates the problems associated with the identification of diseasecausing mutations in individual patients when a plethora of genes may be involved, occasionally simultaneously. In such circumstances, it is important to limit testing to cases where it is clinically indicated and to interpret the results of testing with respect for the insufficient knowledge base of a too detailed clinical inference. 


\section{Financial \& competing interests disclosure}

The authors have no relevant affliations or financial involvement with any organization or entity with a financial interest in or financial conflict with the subject matter or materials discussed in the manuscript. This includes employment, consultancies, honoraria, stock ownership or options, expert testimony, grants or patents received or pending, or royalties.

No writing assistance was utilized in the production of this manuscript.

\section{Bibliography}

1 Hedley PL, Jorgensen P, Schlamowitz S et al.: The genetic basis of long QT and short QT syndromes: a mutation update. Hum. Mutat. 30(11), 1486-1511 (2009).

2 Tester DJ, Will ML, Haglund CM, Ackerman MJ: Compendium of cardiac channel mutations in 541 consecutive unrelated patients referred for long QT syndrome genetic testing. Heart Rhythm 2(5), 507-517 (2005).
3 Napolitano C, Priori SG, Schwartz PJ et al.: Genetic testing in the long QT syndrome: development and validation of an efficient approach to genotyping in clinical practice. JAMA 294(23), 2975-2980 (2005).

4 Schwartz PJ: The congenital long QT syndromes from genotype to phenotype: clinical implications. J. Intern. Med. 259(1), 39-47 (2006)

5 Ackerman MJ: Cardiac causes of sudden unexpected death in children and their relationship to seizures and syncope: genetic testing for cardiac electropathies. Semin. Pediatr. Neurol. 12(1), 52-58 (2005).

6 Berge KE, Haugaa KH, Fruh A et al.: Molecular genetic analysis of long QT syndrome in Norway indicating a high prevalence of heterozygous mutation carriers. Scand. J. Clin. Lab. Invest. 68(5), 362-368 (2008).

7 Larsen LA, Fosdal I, Andersen PS et al.: Recessive Romano-Ward syndrome associated with compound heterozygosity for two mutations in the KVLQT1 gene. Eur. J. Hum. Genet. 7(6), 724-728 (1999).
8 Gouas L, Nicaud V, Berthet $\mathrm{M}$ et al.: Association of KCNQ1, KCNE1, KCNH2 and $S C N 5 A$ polymorphisms with QTc interval length in a healthy population. Eur. J. Hum. Genet. 13(11), 1213-1222 (2005).

9 Priori SG, Schwartz PJ, Napolitano C et al: Risk stratification in the long-QT syndrome. N. Engl. J. Med. 348(19), 1866-1874 (2003).

10 Moss AJ, Shimizu W, Wilde AA et al: : Clinical aspects of type-1 long-QT syndrome by location, coding type, and biophysical function of mutations involving the KCNQ1 gene. Circulation 115(19), 2481-2489 (2007).

11 Makita N, Behr E, Shimizu W et al.: The E1784K mutation in SCN5A is associated with mixed clinical phenotype of type 3 long QT syndrome. J. Clin. Invest. 118(6), 2219-2229 (2008).

12 Hedley PL, Jorgensen P, Schlamowitz S et al.: The genetic basis of Brugada syndrome: a mutation update. Hum. Mutat. 30(9), 1256-1266 (2009).

$13 \mathrm{Ng} \mathrm{SB}$, Turner EH, Robertson PD et al:: Targeted capture and massively parallel sequencing of 12 human exomes. Nature 461(7261), 272-276 (2009). 